\title{
Editorial
}

\section{9th Neuro-oncological Update, Salzburg, 30th January 2009}

\author{
H. Kostron \\ Department of Neurosurgery, Medical University of Innsbruck, Innsbruck, Austria
}

It was of great scientific and practical value to attend this 9th Neuro-oncological Update in Salzburg, focussing on "Malignant Gliomas and the progress made in its therapy". This meeting showed again the importance of interdisciplinary cooperation and supplementation to combat malignant gliomas.

J. Hainfellner, Vienna, opened the discussion by addressing the change of neuropathology induced by molecular diagnostics of brain tumours and its impact on patient management.

Neuroradiology is the most important part for the diagnosis of brain tumours pre- and post-surgery and to evaluate the effect of therapy. This is supported by nuclear medicine. The combination of both modalities is specially important in the light of antiangiogenic therapy and to differentiate real tumour progression from pseudotumour progression. These topics were presented by F. Cartes-Zumelzu and I. Virgolini, Innsbruck. Neurosurgery is the first step in the diagnosis and treatment of brain tumours. The news and progress in this field was reported by H. Kostron, Innsbruck, outlining the importance of preserving the quality of life while resecting most or all of the tumours. The tools are specific preoperative enhanced diagnosis and intraoperative CT, MR and ultrasound as well as image guided surgery by neuronavigation and/or fluorescence guided resection. Intraoperative neurophysiological monitoring or awake operations reduces morbidity significantly. Local intracavitary therapies were addressed by
J. Tonn, Munich, which included BCNU wafers, local radio immunotherapy (J-131 labelled Tanescin) and vector enhanced delivery of fusion proteins. Radiation has the most important impact on the survival of patients with malignant brain tumours. Molecular knowledge changed the paradigm of radiation and re-radiation is now applied with almost the same dose with good effect.

P. Hau, Regensburg, covered the important topic of chemotherapy with Temodal, demonstrating that changing the dose regime for recurrences produce long lasting tumour control. This is even more important in the re-challenge therapy. Antiangiogenic therapy with Avastin ${ }^{\circledR}$ in combination with Temodal ${ }^{\circledR}$ or Irinotecan ${ }^{\circledR}$ has gained wide attention in the treatment of recurrent gliomas resulting in long lasting stable disease. This was reported by J. Pichler, Linz, and R. Mair, Feldkirch.

Finally case reports on various tumour entities such as malignant meningeoma, haemangiopericytoma, and ependymoma, and also cases like a CNS toxoplasmosis were presented and according to "what would you do - what I have done" discussed among the participants in a very active, relaxed and interdisciplinary fashion. These intense case reports have a long-standing tradition and are the favourite part of this neuro-oncological update because of its scientific and practical information. All the participants were grateful for the organization and support of AESCA Pharma, Austria. 